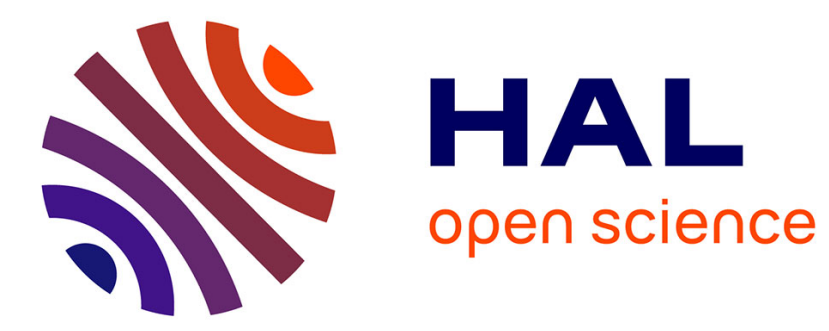

\title{
L'accompagnement des entrepreneurs durant les périodes de doute \\ Patrick Valéau
}

\section{To cite this version:}

Patrick Valéau. L'accompagnement des entrepreneurs durant les périodes de doute. Revue de l'Entrepreneuriat, 2006, 5 (1), pp.31-57. 10.3917/entre.051.0031 . hal-02289192

\section{HAL Id: hal-02289192 \\ https://hal.univ-reunion.fr/hal-02289192}

Submitted on 24 Sep 2019

HAL is a multi-disciplinary open access archive for the deposit and dissemination of scientific research documents, whether they are published or not. The documents may come from teaching and research institutions in France or abroad, or from public or private research centers.
L'archive ouverte pluridisciplinaire HAL, est destinée au dépôt et à la diffusion de documents scientifiques de niveau recherche, publiés ou non, émanant des établissements d'enseignement et de recherche français ou étrangers, des laboratoires publics ou privés. 


\title{
L'accompagnement des entrepreneurs durant les périodes de doute
}

\author{
Par \\ Patrick VALEAU
}

\begin{abstract}
Résumé
Face aux difficultés, les entrepreneurs se doivent de toujours persévérer, mais en pratique la plupart d'entre eux passent par des périodes de doute au cours desquelles leur engagement se fait plus incertain. Leurs accompagnateurs ont alors pour mission de les soutenir pour les aider à trouver un nouveau souffle. Le problème est que ces doutes s'accompagnent souvent d'une détérioration de la relation : lorsque l'entreprise va mal et qu'ils souhaitent s'arrêter, certains entrepreneurs perdent confiance en eux-mêmes ; d'autres remettent en question les compétences de leur accompagnateur. Sur la base de 50 entretiens, cet article expose, tout d'abord, en détail, la nature et les contenus de ces moments de doute. Puis, sur la base d'une étude quantitative menée auprès 127 entrepreneurs accompagnés par l'Association pour le Droit à l'Initiative Economique (ADIE), il analyse les effets de ces doutes sur la relation avec leurs accompagnateurs. Ces résultats aboutissent à un certain nombre de recommandations.
\end{abstract}

\section{Introduction}

Les dispositifs d'accompagnement visent, suivant les cas, à communiquer aux entrepreneurs des informations relatives à l'environnement institutionnel et économique de l'entreprise, à leur transférer des compétences techniques telles que la gestion de trésorerie, à les aider à mûrir leur projet, voire, à leur insuffler un esprit plus entreprenant. Cet article, tout en reconnaissant le caractère incontournable de ces différents aspects, focalise sur le rôle de l'accompagnement en termes de soutien moral et psychologique. Un tel soutien est toujours utile, mais apparaît presque nécessaire durant les périodes de doute. Encore relativement peu étudiée, cette phase du processus entrepreneurial se révèle particulièrement décisive pour la survie de l'entreprise, dans la mesure où elle se manifeste très concrètement par le désir d'abandonner la démarche. Durant ces périodes, les entrepreneurs, surmenés, découragés et souvent déprimés, perdent le recul et perçoivent les difficultés de leur entreprise de façon exacerbée. Dans ce contexte, le rôle de l'accompagnateur est de les soutenir, le temps qu'ils retrouvent leurs motivations et leur persévérance entrepreneuriales.

Le problème est que ces doutes altèrent également la perception que les entrepreneurs ont de leur relation avec leur accompagnateur. Cette dégradation touche les aspects techniques de la relation : l'efficacité perçue de cette dernière, mais également sa dimension affective : son caractère amical. Les accompagnateurs désireux d'apporter aux entrepreneurs un soutien psychologique doivent adapter leurs pratiques de façon à composer avec ces contingences.

Cet article étudie comment accompagner les entrepreneurs durant les phases de doute. La première partie présente tout d'abord une exploration de ces phases restituant un certain nombre de données qualitatives au sein de la littérature existante. Ces données qualitatives proviennent d'entretiens menés auprès de 50 entrepreneurs, et permettent d'emblée de mieux comprendre la nature et les contenus des phases de doute. La deuxième partie expose ensuite l'étude quantitative mesurant les impacts de ces doutes sur la relation avec l'accompagnateur. Ces données quantitatives sont issues d'un questionnaire passé auprès de 127 entrepreneurs accompagnés par l'Association pour le Droit à l'Initiative Economique

Patrick VALEAU - Maître de Conférences

Habilité à Diriger les Recherches FACIREM, IAE de la Réunion

GRAPHE-CLAREE, IAE de Lille

patrick@valeau.com 
(ADIE). Elles évaluent notamment les liens entre le doute et quatre aspects de la relation : l'efficacité, l'amitié, la confiance du suiveur dans l'entrepreneur et la confiance de l'entrepreneur dans son accompagnateur. Nous terminons par une discussion destinée à capitaliser ces premières données sous la forme de quelques conseils pratiques.

\section{Exploration qualitative des périodes de doute}

Clairement, le mot « doute » ne constitue pas un mot clé de la littérature sur l'entrepreneuriat. Le doute se produit néanmoins dans la plupart des cas lors du processus entrepreneurial et cette période pourrait être qualifiée de «phase » de ce processus qui, on le sait, n'est pas linéaire. Dans la littérature en entrepreneuriat, le doute n'a pas encore véritablement fait l'objet de recherches scientifiques et n'est pas ou peu évoqué, les textes insistant plutôt sur la persévérance des entrepreneurs face aux difficultés. C'est pourquoi, suivant une démarche empirico-formelle (Glaser et Strauss, 1967), cet article commence par une exploration de ce phénomène sur le terrain. Cette exploration qualitative nous permet d'emblée d'approfondir le phénomène étudié, de comprendre ces états d'âme pour, dans un second temps, les restituer au sein de la littérature existante.

\subsection{Méthodologie qualitative}

Les phénomènes étudiés étant essentiellement de l'ordre de l'expérience vécue, nos méthodes privilégient tout naturellement l'entretien semi-directif. Nous avons travaillé sur des récits de vie. "L'unité d'analyse » (Yin, 1984) retenue pour cette recherche n'est pas l'entreprise, mais l'individu : son parcours et ses relations avec son entreprise. Nous comparons les expériences et les réactions des uns et des autres et, ce faisant, analysons en profondeur les variables individuelles.

Nous avons retenu, pour cette recherche, 50 entretiens sur un échantillon de 97 comprenant un grand nombre de petites structures. Sans vouloir prendre part au débat sur ce qu'est un entrepreneur (Gartner, Shaver, Gatewood, Katz, 1994) ${ }^{1}$, nous avons préféré écarter un certain nombre de propriétairesdirigeants n'innovant plus du tout. Nous avons gardé ceux continuant d'apprendre, de saisir des occasions d'affaires, de prendre des décisions plus ou moins risquées, impulsant encore leur entreprise. Partant de là, à des fins de comparaison, nous avons constitué un échantillon raisonné en termes de diversité (Morse, 1994), comprenant des individus issus de différentes cultures, ayant créé des entreprises petites ou grandes, opérant dans différents secteurs d'activité (tableau 1).

\begin{tabular}{|l|l|l|c|c|}
\hline Pays / Région & Nombre & Taille & Activités & Ancienneté \\
\hline Région Centre (France) & 12 & \multirow{2}{*}{5 à 100 salariés } & $\begin{array}{c}\text { Agriculture } \\
\text { Industrie } \\
\text { Rervice }\end{array}$ & \\
\cline { 1 - 2 } Réunion (France) & 12 & & & \\
\cline { 1 - 2 } Gloucestershire (Angleterre) & 12 & & & \\
\cline { 1 - 2 } Iles de la Zone Océan & 7 & & & \\
\cline { 1 - 2 } Québec $^{2}$ & 7 & & & \\
\hline
\end{tabular}

Tableau 1 : Composition de l'échantillon

Nous rencontrions les entrepreneurs dans leur entreprise. Nos échanges avec eux commençaient ou se terminaient presque toujours par une visite de cette dernière. Même si l'entreprise n'était pas l'objet de notre étude, cette observation constituait un ancrage important : il permettait un minimum d'expérience partagée des réalités en question. Ces observations servaient à la fois à la réalisation des entretiens et à leur interprétation. Les entretiens proprement dits commençaient toujours par une présentation de l'entreprise : outre les informations ainsi obtenues, c'était une façon de lier connaissance sur un terrain rela- 
tivement concret et objectif. Puis, nous posions à notre interlocuteur une seule et unique question : «Pourrions-nous reprendre votre parcours, en revenant à la première fois où vous avez songé à créer votre propre entreprise ? ». Partant de là, nous suivions notre interlocuteur en lui demandant d'approfondir certaines idées ou en l'interrogeant sur ce qu'il ressentait et voulait à l'époque. Les entretiens partaient ainsi d'éléments relativement techniques pour prendre peu à peu une tournure très personnelle.

La partie la plus délicate de l'entretien était celle concernant les doutes. Comme le montre le tableau 2, les entrepreneurs ressentent, d'ordinaire, une certaine pudeur et, ce faisant, une certaine réticence à évoquer ce qui peut être perçu comme des moments de faiblesse. Les entretiens semi-directifs facilitent cependant la « congruence » et « l'acceptation inconditionnelle » de soi (Rogers, 1961). Dans ce contexte « compréhensif », la plupart de nos interlocuteurs ont évoqué spontanément ces aspects de leur parcours. Dans quelques cas seulement, lorsque notre interlocuteur n'en parlait pas, nous avons demandé : «Avezvous déjà été tenté d'abandonner ? ». Les témoignages ainsi obtenus étaient d'autant plus détaillés, d'autant plus approfondis et d'autant plus justes que ces ressentis étaient, bien souvent, exprimés pour la première fois.

Les critiques relatives à la validité scientifique de ces « récits de vie » sont nombreuses, mais, moyennant quelques délimitations ontologiques et épistémologiques, ils gardent une valeur certaine (Pailot, 2003). On pourra bien évidemment reprocher à cette méthode les risques que l'interviewé ne reconstruise a posteriori les événements. Nous nous intéressons ici à l'expérience vécue, il ne s'agit pas des événements en eux-mêmes, mais de ce que l'individu a pu vivre et ressentir dans ce cadre. La conscience de l'expérience vécue, telle que nous la concevons, passe forcément par une mise en sens qu'elle soit actuelle ou rétrospective. Le sens produit au moment des événements pouvait être tout aussi bien biaisé par la désirabilité pour soi, un refus ou des difficultés à accepter la situation présente. Paradoxalement le sens produit après coup peut s'avérer au moins aussi juste dans la mesure où, avec le recul, l'individu est davantage en mesure d'admettre certaines réalités. Nous pensons également que ces biais peuvent être limités par l'attitude de l'intervieweur, «l'acceptation inconditionnelle » de l'interviewé pouvant l'aider à plus «d'authenticité » (Rogers, 1961). Chaque entretien contribue à cette mise en sens : le fait d'énoncer l'expérience peut ainsi amener des prises de conscience. Il peut stimuler la réflexion et l'élaboration de la pensée sans pour autant modifier les ressentis initiaux.

Nos interprétations opéraient tout d'abord au premier degré : à partir des retranscriptions exhaustives des entretiens, nous tentions de comprendre ce que nos interlocuteurs avaient voulu signifier. Par exemple, par « J'étais fier de mon entreprise », nous comprenions un mélange d'implication affective et normative dans l'entreprise. Par «On ne peut pas douter », nous comprenions simplement une absence de doute.

Dans un second temps, nous abordions ces mêmes discours de façon plus critique : nous analysions l'activité cognitive visant à mettre en sens l'expérience vécue, à travers différentes approches de la communication et, notamment, des éléments de linguistique. Dans l'expression « on ne peut pas douter », nous constations une négation, mais néanmoins une évocation du doute. Nous interprétions cette phrase comme une tentation refoulée : une implication ambivalente entre les ressentis et certaines normes sociales $^{3}$. Nous pouvions ce faisant nuancer nos premières interprétations. Nos interprétations des discours ainsi recueillis ne prétendaient pas à la "vérité » au sens « positiviste du terme », mais à une «vraisemblance » (Guba et Lincoln, 1994 ; Adler et Adler, 1994). Cette double lecture nous permettait une analyse approfondie de chaque entretien.

Dans un troisième temps, nous approfondissions l'analyse en comparant les différents entretiens. Nous avons ainsi relevé, au-delà des différents modes et objets d'engagement, d'importantes similarités concernant notamment les doutes et les changements. Afin de rendre compte de ce processus, nous avons rassemblé et juxtaposé, au sein d'un même tableau, différents extraits d'entretiens. La structure de ce tableau correspond à un modèle relativement simple, s'apparentant à un idéal-type (Weber, 1921) : «Un schéma opératoire permettant de comprendre et de donner un sens à la réalité. Modèle abstrait 
construit à partir de traits caractéristiques et singuliers : il ne s'agit ni de décrire ni de constituer un profil moyen, mais de trouver une structure logique, en ordonnant et en enchaînant des phénomènes isolés et diffus pour former un tableau de pensée homogène ».

La recherche qualitative est un travail d'artisanat (Wacheux, 1996) qui présente un certain nombre de limites, mais aussi d'avantages. Nos catégories et nos remarques ne s'appliquent pas nécessairement à d'autres échantillons, elles ne sont pas forcément statistiquement représentatives. Par la suite, des réplications seront nécessaires afin d'amender et de consolider ce modèle. Cela dit, les propositions ici développées rendent compte de l'ensemble des réalités de notre échantillon : ancrées dans les réalités de terrain, elles saturent les données issues de nos 50 entretiens. Outre ces éléments de " cohérence interne », l'intérêt de ce modèle fut tout d'abord confirmé en triangulant les entretiens ainsi constitués avec sept autres menés par L.J. Filion dans le cadre de la Chaire Entrepreneuriale Rogers-Bombardier. Ces cas supplémentaires constituaient en quelque sorte une première réplication validant la pertinence de ce modèle. Enfin la « vraisemblance » de ce modèle fut établie dans le cadre de la restitution aux intéressés. Le fait qu'ils y trouvent un reflet acceptable, voire éclairant de leur expérience constitue un élément de «validité » important ${ }^{4}$.

\subsection{Méthodologie qualitative}

Nos résultats montrent que presque tous les entrepreneurs passent par des moments de doute durant lesquels l'envie et le plaisir de se comporter de façon entrepreneuriale laissent place au découragement et au désir d'abandonner. Le tableau 2 montre les étapes suivant lesquelles la plupart des entrepreneurs commencent par refuser les doutes (étapes 1,2 et 3), puis les acceptent (étapes 4 et 5), avant de redémarrer (étape 6).

\begin{tabular}{|c|c|}
\hline Thèmes & Illustration \\
\hline $\begin{array}{l}1 . \\
\text { Des moments difficiles }\end{array}$ & $\begin{array}{l}\text { "En } 30 \text { ans de vie de l'entreprise il y a toujours des hauts et des bas. » - «Il y a des } \\
\text { moments où on se dit c'est dur, comment on va finir le mois. » - "J'ai toujours vécu } \\
\text { avec (la précarité), on n’y pense pas à longueur de journée, mais bon... » }\end{array}$ \\
\hline $\begin{array}{l}2 . \\
\text { L'obstination }\end{array}$ & $\begin{array}{l}\text { "Tous les deux on est obstinés, on ferme pas les yeux, on ferme pas les yeux mais } \\
\text { on baisse la tête pour rentrer dedans " - "Il faut être un battant" - "Il faut avoir le } \\
\text { goût de l'aventure.» }\end{array}$ \\
\hline $\begin{array}{l}3 . \\
\text { L'obligation } \\
\text { Donner le change }\end{array}$ & $\begin{array}{l}\text { "Baisser les bras, non on peut pas, il faut faire face à ces périodes là. "- } \\
\text { "Pendant les deux premières années c'était très tendu. On était condamné à réus- } \\
\text { sir! " } \\
\text { "Il faut toujours donner aux gens l'impression que vous êtes le premier, il faut gar- } \\
\text { der la main sinon les gens commencent à douter. " - Il faut aller de l'avant, gar- } \\
\text { der la tête haute, faire voir aux gens que vous êtes là pour gérer l'entreprise. "- } \\
\text { "Ne pas livrer sa perte de confiance en soi ou dans les autres. Se créer un costume } \\
\text { sur lequel on ne perçoit pas de trou. " }\end{array}$ \\
\hline $\begin{array}{l}4 . \\
\text { Lassitude } \\
\text { Décrochage }\end{array}$ & $\begin{array}{l}\text { "Une seconde fois, ça été moi qui ait pensé à arrêter, par lassitude des choses. } \\
\text { Oui !" - "Je me suis assise et me suis mise à rire. " - Par moments tu décroches, } \\
\text { un peu parce que tu ne crois plus, tu en as marre, parce que le lendemain est la } \\
\text { même chose. " - "On sait très bien que le coup de } 4 \text { h du matin est épouvantable } \\
\text { pour l'entrepreneur. Si on pouvait m'assurer de dormir bien tous les matins à } 4 \text { h, } \\
\text { je serais ravie! C'est ça le problème : à ce moment-là, tu ne vivras pas bien, tu ne } \\
\text { vois pas venir les problèmes; ils ne sont pas les bons. Et le lendemain, il fait jour. " }\end{array}$ \\
\hline
\end{tabular}




\begin{tabular}{|c|c|}
\hline $\begin{array}{l}5 . \\
\text { Accepter les doutes et le } \\
\text { changement }\end{array}$ & $\begin{array}{l}\text { "Si on veut continuer, il faut savoir accepter qu'à un moment ou un autre ça n'aille } \\
\text { plus de façon à pouvoir redémarrer avec ce que l'on a d'une manière différente. "- } \\
\text { "En fait vous savez quand on attrape la grippe il y a toujours une période d'incu- } \\
\text { bation et puis après on va mieux. Moi je vis un peu comme ça quand il y a des cho- } \\
\text { ses qui ne vont pas, je serais tenté de dire que je me fous dans un coin pour réflé- } \\
\text { chir. " - Se remettre en question, ça me permet de ne pas vieillir trop, de ne ja- } \\
\text { mais s'asseoir sur des acquis qui ne sont en fait jamais acquis, dans toute situation } \\
\text {...il faut savoir prendre du recul." }\end{array}$ \\
\hline $\begin{array}{l}6 . \\
\text { Des décisions importantes }\end{array}$ & $\begin{array}{l}\text { "On s'aperçoit un jour qu'il y a des choses qui vont plus, donc il faut savoir, } \\
\text { comme je disais tout à l'heure, il faut savoir se mettre dans un coin, se réadapter et } \\
\text { puis prendre la décision qui s'impose. " - "J'ai été très lié à l'entreprise jusqu'à il } \\
\text { y a quelques temps. Mais je m'en suis vraiment détaché. " - "Et puis, j'ai repris } \\
\text { les choses en main, j'ai commencé à déléguer pour me concentrer sur la direc- } \\
\text { tion." }\end{array}$ \\
\hline
\end{tabular}

Tableau 2 : Le processus de remise en question-Extraits de différents entretiens rassemblés par étape

Les entrepreneurs évoquent l'incertitude comme leur condition ordinaire. Ils vivent un quotidien fait d'adversité (extraits 1.). Les témoignages confirment que les entrepreneurs sont bien des individus qui prennent des risques, puis les assument de façon quasi-quotidienne. Face à cette adversité, ils sont fiers de persister. Ils mettent en avant leur ténacité, leur volonté de poursuivre envers et contre tous (extraits 2.). Cela dit, défier cette adversité leur donne des sentiments partagés entre un certain plaisir, un peu d'orgueil et beaucoup de stress. Les entrepreneurs présentent également cette persévérance comme une nécessité, une obligation : l'activité de l'entreprise ne leur laisse guère le choix. Ils sont pris dans l'action (Extraits 3). Au-delà, le doute apparaît comme un interdit social (extraits 3. suite).

Les remises en question des extraits 4 sont d'un tout autre ordre : dans ces extraits, les entrepreneurs évoquent de vrais moments de doute : ceux au cours desquels ils ne savent plus s'ils veulent encore continuer. Ces doutes sont rarement exprimés d'emblée, en début d'entretien, les entrepreneurs préférant, au moins dans un premier temps, évoquer leurs succès passés et à venir. Cette réserve s'explique également par le fait que ces ressentis ne sont pas toujours complètement analysés : ils restent souvent à l'état de sentiments flous, de contradictions et de refoulement. L'entretien accompagne la réflexion des entrepreneurs : avec la confiance et la neutralité que peuvent offrir les techniques rogériennes, certains peuvent parler, voire, dans certains cas, prendre conscience de ces doutes, pour la première fois.

Ces périodes de questionnement amènent les entrepreneurs à réfléchir de nouveau sur eux-mêmes, à se retourner sur le chemin parcouru. Suivant cette introspection, ils regardent leurs erreurs, prennent conscience de leurs émotions et de leurs sentiments, se « laissent aller » à "s'écouter » (Extraits 4 et 5). Après un moment de submersion et de «lâcher prise ", les entrepreneurs remettent en ordre et en sens les évènements et les émotions de leur passé. Les périodes de doutes débouchent souvent sur des décisions majeures (Extraits 6), comme, par exemple, une séparation avec un associé ou l'abandon de certains marchés. Dans la plupart des cas, ces décisions actualisent les liens entre le projet personnel et le projet d'entreprise (Bruyat, 1994 ; Verstaete, 1999, 2003). Ces décisions marquent généralement pour l'entreprise le début d'une nouvelle ère fondée sur un nouvel engagement des entrepreneurs : sur un second souffle mieux ajusté.

Les données qualitatives ainsi analysées permettent de mieux « sentir » les caractéristiques et les contenus possibles de ces périodes : le doute peut être défini comme un état d'indétermination, une hésitation entre poursuivre l'entreprise et abandonner, le plus souvent dans l'idée de devenir salarié. C'est un état qui peut, dans certain cas, se prolonger, mais reste a priori transitoire, il précède une décision de fait entre l'une ou l'autre des alternatives envisagées.

Le doute est lié à des états psychologiques complexes, qui plus est relativement variés d'un entrepreneur à l'autre. Ses origines peuvent mêler de l'insatisfaction et de la déception par rapport aux expériences vécues. Ce doute peut aussi venir des inquiétudes des entrepreneurs concernant leurs capacités à réussir ou leurs chances de voir leurs efforts récompensés. Au delà, le doute est, presque toujours, associé à de 
la lassitude, du découragement voire des formes de dépression entrainant des tendances au pessimisme. Suivant un cercle vicieux, ce pessimisme alimente et exacerbe les insatisfactions et les inquiétudes.

Le fait d'envisager la possibilité de tout arrêter, de faire autre chose, de devenir salarié, crée une brèche dans la persévérance entrepreneuriale, mais, cette dernière ne disparaît complètement pour autant. Le doute se caractérise par la cohabitation d'états, de sentiments et de motivations contradictoires : les entrepreneurs envisagent d'arrêter et, en même temps, compte tenu de leurs engagements psychologiques, sociaux et, bien évidemment économiques, ne peuvent s'y résoudre. Ces sentiments contradictoires participent à un état de confusion durant lequel les entrepreneurs perdent une grande partie de leurs énergies. Leur volonté, si cruciale pour leur entreprise, se dilue.

Ces premières explorations des périodes de doute nous permettent d'apporter quelques nuances par rapport à certaines théories en entrepreneuriat. Cet article réintroduit le doute au cœur d'un processus à l'issue duquel la persévérance finit souvent par reprendre le dessus, mais ne peut être tenue pour acquise. Si la plupart des entrepreneurs se reprennent, finissent par remettre de l'ordre dans leurs idées et repartent, d'autres s'arrêtent pour de bon.

\subsection{Restitution des périodes de doute au sein de la littérature sur l'entrepreneuriat}

Nous nous efforçons à présent de conceptualiser cette problématique du doute en la reliant aux théories existantes. Nous revenons tout d'abord sur l'activité entrepreneuriale en retenant comme élément fondateur «l'impulsion » donnée par les entrepreneurs, c'est elle, en effet, que les doutes risquent de remettre en question. Nous reprenons ensuite un certain nombre de textes introduisant des sentiments plus ou moins proches de ceux étudiés. Enfin nous abordons les activités d'accompagnement en mettant en relief leurs aspects psychologiques.

\subsection{1. «L'impulsion » entrepreneuriale}

Une grande partie des recherches en entrepreneuriat étudie ce que " fait » effectivement l'entrepreneur (Gartner, 1988). Dans ce sens, l'entrepreneuriat peut être défini comme un ensemble d'activités associées à la création et au développement d'une entreprise. Ainsi, l'entrepreneur est avant tout un innovateur (Schumpeter, 1935) et un preneur de risques (Mill, 1848, Say, 1803 ; Knight, 1921) sans cesse à la recherche de nouvelles opportunités d'affaires à exploiter (Cantillon, 1757 ; Say, 1803, Kirzner, 1993). Ces approches construites par les auteurs classiques des siècles passés ont, pendant longtemps, constitué le cœur de la littérature sur l'entrepreneuriat. Pour certains, ces activités caractérisent la phase de création (Bygrave \& Hofer, 1991 ; Gartner, Bird \& Starr, 1992 ; Gartner \& Gatewood, 1992 ; Davidson, Low and Wright, 2001) : avant que l'entreprise ne soit complètement établie (Weick, 1979 ; Gartner, Bird \& Starr, 1992). Pour d'autres la phase entrepreneuriale se poursuit aussi longtemps que l'entrepreneur saisit de nouvelles opportunités (Filion, 1997 ; Shane \& Vankatamaran, 1997 ; Ucbasaran, Westhead and Wright, 2001) et développe son entreprise (Carland, Hoy, Boulton \& Carland, 1984 ; Davidson \& Wiklund, 2001). Pour ces auteurs, le défi de l'entrepreneuriat consiste à perpétuer l'entreprise au moins autant qu'à la créer (Naffziger, Kuratko, Hornsby, 1994). Dans ce sens, la phase entrepreneuriale se poursuit aussi longtemps que l'individu « impulse » l'entreprise, cette impulsion incluant tour à tour la dynamique créée pour que le phénomène se manifeste, puis les forces nécessaires pour que celui-ci persiste (Verstraete, 2002, 2003). Partant de là, de nombreuses contributions se sont intéressées aux qualités communes aux individus à l'origine de ces activités. Brockaus \& Horwitz (1985) et Verstraete (1999) résument ces recherches empiriques : l'entrepreneur est un individu caractérisé par le besoin de réalisation de soi ; l'indépendance et un locus de contrôle interne. Ces recherches se poursuivent encore aujourd'hui : certaines confirment la spécificité de la personnalité des entrepreneurs (ex. Johnson, 1990) ; d'autres montrent que ces derniers ne sont pas forcément très différents des non-entrepreneurs (Vesalainen et Pihkala, 1999 ; Gartner, Shaver and Gatewood, 1994) ; des auteurs comme Shaver (1995) remettent, ce faisant, en cause certains mythes concernant les entrepreneurs. Face aux difficultés d'aller plus loin dans la mise à jour de ces caractéristiques communes et distinctives des entrepreneurs, de plus en plus de textes mettent en avant la diversité des entrepreneurs. 
La recherche francophone relie ce que « fait » et ce qu' « est » l'entrepreneur dans le cadre de modèles fondés sur une distinction entre l'entrepreneur et l'entreprise : leurs logiques sont interdépendantes mais peuvent être différentes. Verstraete (2002) modélise l'entrepreneuriat par un triple regard : cognitif (la vision, la réflexivité et l'apprentissage de l'acteur), praxéologique (l'action d'entreprendre) et structural (interactions et imbrication avec de multiples contextes), pour comprendre le phénomène liant l'entrepreneur et l'organisation (processus et résultat) qu'il impulse. Bruyat (1994) met également en perspective les interactions dialogiques entre la condition de l'entrepreneur et les innovations liées à son projet d'entreprise. Ces recherches permettent de mieux comprendre l'influence de l'entrepreneur : sa personnalité, sa vision, mais aussi son moral sur le développement de l'entreprise. Suivant cette perspective, le doute constitue une fluctuation, une confusion et une altération des motivations sur lesquelles se fonde l'impulsion du processus entrepreneurial.

\subsubsection{Des stress aux doutes}

Pour Shaver (1995), les vrais entrepreneurs sont ceux qui persistent à travers tous les défis, alors que les individus qui auraient essayé une fois, qui auraient échoué puis auraient jeté l'éponge seraient des «nonentrepreneurs ». Ces hypothèses se trouvent largement vérifiées par les résultats des recherches menées par Cardon et McGrath (1999) : dans leur article « Quand les choses se gâtent... pour une psychologie de l'échec et de la remotivation des entrepreneurs ${ }^{5}$, ils constatent que les individus attribuant leurs difficultés à la quantité d'efforts fournis se remotivent plus facilement que ceux remettant en cause leurs circonstances ; face aux contre-performances, les premiers redoublent d'efforts. Ces réflexions rejoignent celles de Simon, Houghton et Aquino (2000) pour qui l'impression de contrôler les choses qui caractérise les entrepreneurs favoriserait la persévérance face aux incertitudes relatives à la survie de l'entreprise et face aux coups durs. Ces réflexions introduisent certaines nuances mais confirment globalement le mythe entrepreneurial (Shaver, 1995) : l'entrepreneur connaît des coups durs, mais se relève très vite. Afzalur (1996) reformule ainsi cette idée : « les entrepreneurs sont fascinants. Ils alternent entre l'extase du succès et les affres de l'échec, ils rebondissent pour revivre à nouveau. Ils ont un fort besoin d'excitation et de risques.» Cette première série de références évoque les coups durs, mais passe très rapidement sur les moments de doute. Suivant nos données qualitatives, effectivement la plupart des entrepreneurs repartent, mais ce rebondissement ne constitue jamais une simple formalité, il ne saurait être tenu pour acquis.

Différents auteurs décrivent les stress liés à l'engagement entrepreneurial. Pour Boyd et Gumper (1983) le surmenage est inévitable, il constitue un corollaire de cette expérience. Pour Adebowale (1994) le temps passé dans l'entreprise, l'immersion dans l'activité expliquent en partie ce stress, mais il accuse également un besoin d'accomplissement et des ambitions démesurés qui amènent parfois l'entrepreneur à « fixer la barre trop haut » et à vouloir « en faire trop ». Afzalur (1996) évoque des symptômes tels que l'anxiété, la dépression et l'irritabilité. Pour ces deux auteurs, les entrepreneurs peinent à gérer ce stress à cause du manque de soutien. Adebowale (1994) invite les entrepreneurs à en prendre conscience. Sous le terme de «stress », les différents auteurs semblent signifier des contenus relativement différents. Au départ, le stress correspond à un état de tension destiné à rassembler et focaliser les énergies contre l'adversité. La persistance de ce stress peut, à la longue, générer le surmenage et la dépression. Les états évoqués par Boyd et Gumpert (1983), Adebowale (1994) et Afzalur (1996) semblent correspondre aux étapes 1,2 et 3 du processus décrit dans le cadre du tableau 2 de notre exploration qualitative : les entrepreneurs supportent le surmenage, le stress ressenti constitue une réaction physique et psychique qui, jusqu'à un certain point, renforce leurs énergies. Ils n'envisagent pas encore, à ce stade, d'arrêter.

Certains auteurs évoquent, pour leur part, des sentiments d'insatisfaction. Pour Naffziger, Hornsby, Kuratko (1994), les entrepreneurs continuent leur entreprise dans la mesure où leurs attentes sont satisfaites. Nos explorations qualitatives montrent des situations plus compliquées : les entrepreneurs peuvent avoir envie d'arrêter, mais hésiter à se désengager compte tenu de leurs investissements antérieurs (Staw, 1976 ; Beauvois et Joule, 1987 / travaux menés dans d'autre domaines). Une telle situation peut, 
dans certains cas, être ressentie comme aliénante, les entrepreneurs se sentant alors comme prisonniers de leur entreprise. D'une façon générale, le doute est rarement vécu comme un état serein durant lequel les entrepreneurs examineraient posément et rationnellement les avantages et les inconvénients de continuer ou d'arrêter.

Le doute, tel que nous l'avons découvert dans le cadre de nos explorations qualitatives, est un état complexe, mêlant les différents éléments introduits par les auteurs cités ci-dessus, mais ne pouvant être limité à aucun d'entre eux. Il se manifeste très concrètement par une incertitude sur la suite à donner à l'entreprise : l'entrepreneur envisage l'éventualité d'arrêter. Absents de la littérature scientifique, certains sentiments liés au doute sont exprimés de façon parfois très explicite dans la presse professionnelle. Le texte de Stevens (1991) «Rechargez vos batteries entrepreneuriales » évoque tout d'abord un phénomène d'usure : «Malheureusement, si vous êtes comme beaucoup d'entrepreneurs, ce qui a commencé comme une aventure n'est maintenant à peine plus qu'un travail. Avec les années, l'excitation et le défi de conduire sa propre entreprise ont laissé la place à la routine et à l'ennui. ». Stevens aborde ensuite très précisément ce que nous considérons comme la principale composante du doute : «Vous avez envie de laisser tomber. Vous êtes prêt à vous débarrasser de ce poids que vous appelez votre entreprise. » Le doute correspond à un état d'indétermination et d'hésitation entre poursuivre l'entreprise et abandonner le plus souvent dans l'idée de devenir salarié. Le stress, l'insatisfaction et la déprime alimentent ce processus sous la forme d'un cercle vicieux.

Le doute participe à un processus qui, au-delà des potentialités des situations et des acteurs, demeure relativement indéterminé : d'un point de vue subjectif, les entrepreneurs hésitent ; d'un point de vue objectif, l'issue de ce processus reste incertaine. Partant de là, nous pensons que l'aboutissement de certaines aventures entrepreneuriales peut être influencé par une aide extérieure comme celle que peuvent apporter les accompagnateurs.

\subsubsection{L'accompagnement psychologique des entrepreneurs}

L'accompagnement est une problématique en phase de « durcissement » (Stengers, 1987) ${ }^{6}$ : le nombre d'articles scientifiques sur le sujet reste encore relativement limité, mais croît très rapidement. La réflexion sur l'accompagnement peut, par ailleurs, être reliée à des problématiques plus anciennes relatives à la formation (ex. Béchard, 1998; Saporta et Verstraete, 2000) et au conseil (ex. Cullière, 2004) : dans tous les cas, la question reste de savoir que communiquer à des individus désireux de se lancer en affaires. Cela dit, suivant certaines acceptions, l'accompagnement prend un sens plus large : au départ focalisé sur les aspects technico-économiques de la création d'entreprise, la plupart des auteurs évoquent le développement de savoir-être entrepreneuriaux.

Dokou, Gasse, Abiassi et Camion (2004) définissent l'accompagnement comme un ensemble de pratiques destinées à combler les insuffisances stratégiques des PME. Ils étudient l'influence de l'accompagnement dans la surveillance de l'environnement, dans l'accès aux ressources locales et dans la gestion des risques. Sammut (2003) reprend la définition de Johannisson (1991) : l'accompagnement doit permettre aux créateurs de faire mûrir leur projet en leur offrant un guide de réflexion pour l'action, il facilite l'accès à l'information et à la connaissance. De façon plus générale, l'accompagnement doit, selon elle, permettre aux entrepreneurs de penser et de gérer la complexité du processus : il s'agit de les aider à décliner leur stratégie dans le management quotidien (Verstraete, 1997 ; Sammut 2003). Ces objectifs passent, au départ, par le transfert d'informations et de connaissances technico-économiques, mais peuvent également impliquer des éléments de savoir-être.

Ainsi, certains auteurs évoquent, à propos de l'accompagnement, la sensibilisation à certaines valeurs associées à un état d'esprit entrepreneurial. Dokou (2002) étudie, par exemple, dans quelle mesure la communication de ces valeurs pourrait avoir un impact sur la saisie d'opportunités. Alvarez (1993) aborde la question de façon plus critique en parlant d'idéologie. De façon plus générale, on évoque de plus en plus un risque d'ingérence de l'accompagnateur dans le projet de l'entrepreneur (Congrès de l'Académie de l'Entrepreneuriat, 2005). Face à ces possibles dérives, Verstraete (1997) et Valéau (2001) soulignent la nécessité de respecter les entrepreneurs, ils invitent les formateurs et les accompagnateurs à prendre en compte leurs motivations, leurs univers cognitifs et les facteurs que ceux-ci jugent les plus 
importants pour leur réussite. Partant de là, stimuler les capacités entrepreneuriales des individus signifie notamment les amener à élaborer leur vision (Verstraete, 1997, Saporta et Verstraete, 2000).

Cette approche élargie de l'accompagnement peut être rapprochée du conseil en orientation ou de certaines conceptions des bilans de compétences : il s'agit d'accompagner l'individu dans la maturation et le développement de son projet. Comme le soulignent Bruyat (1994), Verstrate (1999) et Valéau (2001), le projet d'entreprise est très lié au projet individuel. Dans ce sens, nous pensons que l'accompagnement passe par une approche plus globale de la personne incluant son développement personnel. Ces approches globales se retrouvent dans les pratiques et le concept anglosaxon de « counseling » : Priels (2004) résume le "counseling » comme une forme d'accompagnement fondé sur la compréhension de la personnalité, des représentations personnelles, du style de vie et des rôles sociaux de l'individu, autrement dit sur une appréhension de sa personne toute entière. La démarche du « counseling » s'appuie sur la clarification des solutions que la personne peut trouver en elle-même pour résoudre les difficultés devant lesquelles elle se trouve. Le " counseling » vise la facilitation du développement humain, du potentiel d'évolution et de changement. Cette approche utilise souvent les techniques de l'entretien développé par Rogers (1961). Suivant cette ouverture au " counseling ", l'accompagnement de l'entrepreneur peut prendre la forme d'un soutien moral et psychologique à la création mais également tout au long des développements et redéveloppements du processus entrepreneurial. .

Qu'il prenne une dimension psychologique ou qu'il reste essentiellement technique, l'accompagnement s'apparente à une "relation d'aide ». Bares et Muller (2002) évoquent les conflits d'intérêts possibles entre l'accompagnateur et l'entrepreneur. Castra (2003) ${ }^{7}$ et Valéau (2004) ${ }^{8}$ évoquent les jeux de condescendance et de stigmatisation vers lesquels ceux qui sont sensés aider peuvent, parfois, faire dériver la relation d'aide : ceux qui veulent aider dans des rôles de " sauveurs » ont parfois tendance à enfermer leurs interlocuteurs dans des rôles infantilisants contraires à leur autonomisation. A l'inverse, la relation d'aide peut être entravée par les entrepreneurs eux-mêmes : Cullière (2004) évoque ainsi des résistances liées à la perception de la légitimité des intervenants ou à une incompréhension du dispositif. Sammut (2003) évoque également des jeux comme des demandes d'expertise sans accès aux informations de l'entreprise. Suivant ces perspectives, la relation d'accompagnement apparaît complexe et ambiguë.

Cet article étudie les besoins et les difficultés d'une relation d'aide psychologique aux entrepreneurs en phase de doute. Cette exploration nous ayant permis de mieux comprendre la complexité et les ambiguïtés de l'état d'esprit des entrepreneurs dans ces moments, la partie suivante tente de mesurer les impacts des doutes sur les relations avec les accompagnateurs s'efforçant de les soutenir.

\section{Etude quantitative des doutes et de leurs implications sur les rela- tions avec l'accompagnateur}

Les méthodes qualitatives permettaient de comprendre et d'intégrer l'interpénétration des phénomènes liés au doute, en restituant ces hésitations quant aux suites à donner à l'aventure entrepreneuriale, en lien avec les stress, les insatisfactions et les «blues ». Le passage à la quantification se révèle à bien des égards plus délicat. Nous fondons notre mesure de ce concept sur son indicateur le plus manifeste, le plus directement observable et le plus intrinsèquement lié à cet état d'âme : le fait d'envisager la possibilité d'arrêter l'aventure. Au-delà des causes, nous considérons le doute comme une période durant laquelle les motivations et l'implication sont, pour différentes raisons, remises en question. Cette étude quantitative revient tout d'abord sur les liens, voire les imbrications possibles, entre le fait d'envisager d'arrêter et l'état des motivations à continuer. Partant de là, elle mesure l'impact du doute sur la perception des relations avec les accompagnateurs. 


\subsection{Méthodologie quantitative}

La population étudiée était celle de l'Association pour le Droit à l'Initiative Economique (ADIE) : des bénéficiaires du RMI et des chômeurs désireux de monter leur propre entreprise. Cette population, plus ciblée que dans le cadre des entretiens semi-directifs, vit un processus entrepreneurial relativement identique à celui suivi par les autres populations étudiées, passant notamment par les mêmes périodes de doute. L'ADIE propose à ces entrepreneurs un prêt et un accompagnement. Le questionnaire que nous leur avons soumis combinait les préoccupations de l'ADIE concernant l'impact de son action en termes d'insertion sociale et économique et les problématiques de cette recherche sur l'accompagnement.

Une première série de huit questions intitulée " Vos motivations et vos expériences » portait sur l'état d'esprit de l'entrepreneur. Par exemple, s'était-il lancé en affaires par défi ou par nécessité ? Les questions suivantes concernaient ses compétences et ses expériences antérieures. Cette partie se terminait par une question reprenant des items d'un questionnaire d'internalité (Pasquier et Lucot, 1999) : «8. Pour réussir une entreprise, pensez-vous que : Il faut beaucoup de chance - Il faut beaucoup de préparation - Il faut certaines qualités personnelles - Il faut connaître des gens influents - Il faut beaucoup d'efforts »

Une deuxième série de quatorze questions intitulée : "Votre entreprise », reprenait un ensemble d'indicateurs relatifs au chiffre d'affaires, à la santé de l'entreprise et à la situation financière de l'entrepreneur. Parmi ces questions se trouvaient, par exemple, les suivantes : «10. Globalement la santé de votre entreprise vous semble-t-elle bonne ? » «14. Actuellement, quels types de difficultés rencontrez-vous dans votre entreprise : financières ? Vente ? Approvisionnement ? Organisation ? Personnelles ?» «17. Les revenus dégagés de votre entreprise sont-ils suffisants pour répondre aux besoins de votre ménage ?» «21. Avec la création de votre entreprise, votre situation financière s'est-elle améliorée ?»

Une troisième série de treize questions, intitulée « Les prêts dont vous avez bénéficié », abordait de façon très détaillée les prêts et les primes obtenus par l'entrepreneur, ainsi que son insertion bancaire avant et après la création de l'entreprise. L'insertion bancaire étant l'une des vocations de l'ADIE, ces données n'ont pas encore été exploitées et concernent des problématiques plus spécifiques à cette association.

Une quatrième série de quatre questions, intitulée « Le suivi dont vous avez bénéficié » portait sur la relation avec l'accompagnateur : ses modalités et sa fréquence, les besoins de l'entrepreneur et la qualité des réponses obtenues. Ces questions seront intégrées par la suite. Dans le cadre de cet article, nous avons privilégié les réponses aux questions suivantes : « 41 . Comment voyez-vous votre relation avec votre suiveur ?Diriez-vous que votre relation était efficace - Diriez-vous que votre relation était amicale - Diriez-vous que vous aviez confiance dans votre suiveur - Pensez-vous que votre suiveur avait confiance en vous "

Une cinquième série de questions portait sur la satisfaction des entrepreneurs aujourd'hui. Par exemple, la question 42 était la suivante : "La création de votre entreprise a-t-elle finalement été une expérience positive ? Vous a-t-elle donné plus confiance en vous ? Vous a-t-elle rendu(e) plus optimiste ? Vous a-telle tranquillisé(e) ? Vous a-t-elle rendu(e) plus entreprenant(e) ? A-t-elle rendu votre vie plus intéressante ? Vous a-t-elle permis d'être mieux respecté(e) ? »

La question 43 reprenait, quant à elle, quelques items du questionnaire sur l'implication d'Allen et Meyer (1997) : « Aujourd'hui, pensez-vous : Je suis fier(fière) d'avoir créé mon entreprise ; Je me sens affectivement attaché(e) à mon entreprise ; Il ne serait pas bien d'arrêter mon entreprise, même si j'y trouvais avantage ; Arrêter mon entreprise aurait plus d'inconvénients que d'avantages ; Je continue mon entreprise parce que je ne vois pas d'autres possibilités. Les trois premiers items mesuraient les attachements normatifs et affectifs à l'entreprise, les deux derniers évoquaient l'implication continue (Allen et Meyer, 1997), autrement dit la relative irréversibilité des processus d'investissement engagés (Staw, 1976).

La dernière question était la suivante "Pour l'avenir, préféreriez-vous (re)trouver un emploi salarié ?».L'une des questions à laquelle l'analyse des données devait répondre était de savoir si cet indica- 
teur pouvait ou non être associé aux items liés à la satisfaction et à l'implication pour former un construit homogène ou s'il fallait, comme nous en faisions l'hypothèse, les considérer comme des phénomènes liés mais de nature distincte.

Le questionnaire était construit de façon à commencer par des questions relativement factuelles en s'orientant vers des questions plus sensibles impliquant davantage d'opinions et de ressentis. Suivant les théories notamment décrites par Beauvois et Joule (1987), les répondants se trouvaient peu à peu engagés dans la démarche.

Les questionnaires ont été passés directement, par téléphone, avec l'aide de trois étudiants de Maîtrise en Sciences de Gestion, durant le mois de Janvier 2005 grâce aux coordonnées communiquées par l'ADIE. Il s'agissait des entrepreneurs accompagnés au cours des cinq dernières années. La quasi-totalité des entrepreneurs contactés ont accepté de répondre à l'ensemble des questions posées, ce taux particulièrement élevé s'explique sans doute par le statut des étudiants donnant sans doute à la démarche un caractère plus neutre. Les questions étaient toujours livrées de façon standard mais pouvaient dans un second temps être expliquées en fonction des questions des interlocuteurs. Par exemple, dans les cas où ces derniers n'avaient jamais eu d'emploi salarié, la question concernant la possibilité de retrouver un emploi salarié pouvaient être changée en «trouver un emploi salarié». Les résultats obtenus sont le fruit d'une implication très active de la part des étudiants ayant participé à ce projet.

La principale difficulté résidait en fait dans les changements de coordonnées et d'adresses. Ce phénomène fut surtout ressenti pour les entrepreneurs ayant d'ores et déjà abandonné, l'échantillon obtenu $(\mathrm{n}=30)$ se révélant trop restreint pour l'analyse statistique. Au final, nous avons pu travailler sur la base de 127 entrepreneurs encore en activité. Compte tenu de la variance constatée au niveau des difficultés rencontrées, ces entrepreneurs pouvaient, suivant les cas, être considérés comme ayant plutôt réussi ou plutôt en situation d'échec.

Afin d'optimiser les possibilités d'analyse, la plupart des questions étaient assorties d'échelles quantitatives de 1 à 7 , les réponses ainsi obtenues pouvaient être considérées comme des variables continues. Les traitements : corrélations bilatérales de Pearson, corrélations partielles, alpha de Cronbach et régressions, ont été exécutés avec l'aide du logiciel SPSS.

Une question subsidiaire de cette étude concerne les caractéristiques et les éventuelles spécificités de la population étudiée. Il serait, par la suite, intéressant de reconduire cette recherche auprès d'autres populations. Il s'agirait, ce faisant, de savoir si les connaissances présentées dans le cadre de cet article sont liées à la situation d'exclusion ou à la démarche entrepreneuriale. Nous faisons l'hypothèse que la population ici étudiée n'a pas forcément un potentiel entrepreneurial plus faible que la population salariée : les chômeurs et les bénéficiaires du RMI développent souvent des activités informelles qui, mises à part les normes juridiques, peuvent être considérées comme des démarches pré-entrepreneuriales. Cela dit, ils créent leur entreprise, sans doute plus souvent que les salariés, par nécessité. Les résultats montrent que, au sein même de cette population, ceux qui ont créé par défi ou opportunité sont moins enclins au doute. La question consiste à savoir s'ils peuvent, chemin faisant, devenir de "véritables » entrepreneurs. Une partie de la réponse se trouve, selon nous, dans les pratiques d'accompagnement. Le doute est aussi l'histoire d'une transformation profonde de soi, un changement d'identité sociale mais aussi et surtout psychologique. Cette transformation rouvre la perspective suivant laquelle on ne nait pas entrepreneur, mais on le devient.

\subsection{Résultats : des doutes au-delà de la santé de l'entreprise}

Les données quantitatives recueillies dans le cadre du questionnaire adressé aux entrepreneurs suivis par l'ADIE complètent nos données qualitatives, nous commençons par les données sur le doute proprement dit, avant d'analyser ses effets sur les relations entrepreneur-accompagnateur.

2.2.1. Doute, insatisfaction et implication

Les données quantitatives montrent tout d'abord que le doute et l'envie d'arrêter l'entreprise s'accompa- 
gnent généralement d'autres symptômes : les entrepreneurs posent un jugement globalement négatif sur leur parcours, ils ne sont pas tranquilles (tableau 3). Le sens de la causalité reste ici à définir : une première interprétation envisagerait l'envie d'arrêter comme une conséquence de l'insatisfaction, une seconde interprétation, compte tenu des données qualitatives, montrerait que le processus de doute génère des formes de dépression renforçant les jugements négatifs.

\begin{tabular}{|c|c|c|}
\hline & $\begin{array}{c}\text { Expérience } \\
\text { Positive }\end{array}$ & Tranquillité \\
\hline $\begin{array}{l}\text { Doute / envie } \\
\text { d'abandonner }\end{array}$ & $-0,2^{*}$ & $-0,23^{* *}$ \\
\hline
\end{tabular}

Tableau 3. Corrélations entre les doutes et la satisfaction

** La corrélation est significative au niveau 0.01 (bilatéral)

* La corrélation est significative au niveau 0.05 (bilatéral)

\begin{tabular}{|c|c|c|}
\hline & $\begin{array}{c}\text { Expérience } \\
\text { Positive }\end{array}$ & Tranquillité \\
\hline $\begin{array}{l}\text { Doute / envie } \\
\text { d'abandonner }\end{array}$ & $-0,2^{*}$ & $-0,23^{* *}$ \\
\hline
\end{tabular}

Tableau 4. Corrélations entre le doute et l'implication

Les données quantitatives montrent tout d'abord que le doute et l'envie d'arrêter l'entreprise s'accompagnent généralement d'autres symptômes : les entrepreneurs posent un jugement globalement négatif sur leur parcours, ils ne sont pas tranquilles (tableau 3). Le sens de la causalité reste ici à définir : une première interprétation envisagerait l'envie d'arrêter comme une conséquence de l'insatisfaction, une seconde interprétation, compte tenu des données qualitatives, montrerait que le processus de doute génère des formes de dépression renforçant les jugements négatifs.

Le tableau 4 montre des corrélations avec un des items de l'implication continue. Ces données confirment l'exploration qualitative : les entrepreneurs qui doutent continuent parce qu'ils ne voient pas d'autres solutions : ils envisagent d'arrêter mais se trouvent engagés dans un processus dont ils ne peuvent facilement se défaire. La corrélation avec l'item d'implication morale selon lequel « il ne serait pas bien d'arrêter " peut paraître, à certains égards, plus surprenante. Nous l'interprétons comme une confirmation de l'exploration qualitative concernant les sentiments contradictoires qui caractérisent la phase de doute : les entrepreneurs sont tentés d'arrêter et, en même temps, ils gardent des engagements affectifs et normatifs. Ces corrélations indiquent une implication confuse dans le cadre de laquelle le pouvoir, le devoir et le vouloir se télescopent. Ceux qui ne doutent pas n'ont, de leur côté, pas besoin d'entrer dans ce type de considérations.

Ces liens entre le doute et les deux items de satisfaction, puis avec les deux items d'implication ne permettent cependant pas d'envisager une consolidation de ces mesures sous la forme de construits homogènes et cohérents : leurs alpha de Cronbach respectifs $(0,61$ et 0,43$)$ ne sont pas suffisants. Le doute sur la suite à donner à l'entreprise constitue la manifestation d'un phénomène dont la complexité dépasse, pour l'instant, les indicateurs développés dans le cadre de notre questionnaire et les relations, jusque là, établies entre eux. Faute de mieux, nous conservons le fait d'envisager d'arrêter l'entreprise pour devenir salarié comme principal indicateur du doute.

\subsubsection{Doute, santé de l'entreprise et potentiel entrepreneurial}

Le doute est souvent lié au succès de l'entreprise : de façon évidente, les entrepreneurs sont plus enclins à poursuivre lorsque la santé globale de l'entreprise est bonne que lorsqu'ils rencontrent des difficultés financières qui rapidement mettent en danger leurs finances personnelles. Cela dit, ces corrélations ne sont pas totales : il arrive de façon relativement fréquente que les entrepreneurs aient envie d'arrêter alors que l'entreprise marche bien et, inversement, conformément à certains arguments de la littérature scientifique, les entrepreneurs peuvent vouloir continuer alors que l'entreprise est en difficulté. La variable personnalité semble, jusqu'à un certain point, modérer les effets de la santé de l'entreprise sur 
l'envie d'arrêter. La corrélation partielle présentée dans le tableau 7 permet de neutraliser ces derniers : à santé égale, ceux qui ont démarré pour saisir une opportunité sont moins enclins à arrêter. Les effets de la création par défi $(-0,16, p<0,65)$ sont également proches du seuil de significativité requis $(p<0,5)$. Conformément à certaines théories (ex. Johnson, 1990 ; Shaver, 1995), nos données montrent qu'une personnalité au départ plus entrepreneuriale peut jouer un rôle modérateur sur les doutes et le désir d'arrêter face aux difficultés de l'entreprise. Partant de là, ce sont plutôt les " autres ", ceux qui ont créé par nécessité plus que par défi, qui ont le plus besoin du soutien d'un accompagnateur.

\begin{tabular}{|c|c|c|c|}
\hline & Santé de l'entreprise & $\begin{array}{c}\text { Difficultés } \\
\text { Financières }\end{array}$ & $\begin{array}{c}\text { Finances } \\
\text { Personnelles }\end{array}$ \\
\hline $\begin{array}{c}\text { Doute / envie d'aban- } \\
\text { donner }\end{array}$ & $-0,26^{* *}$ & $0,28^{* *}$ & $-0,3^{* *}$ \\
\hline
\end{tabular}

Tableau 5. Corrélation entre le doute et la santé de l'entreprise

\begin{tabular}{|c|c|c|}
\hline & Création / Opportunité & $\begin{array}{c}\text { Création } \\
\text { par défi }\end{array}$ \\
\hline Doute / envie d'abandonner & $-0,25^{* *}$ & $-0,18^{*}$ \\
\hline
\end{tabular}

Tableau 6. Corrélation entre le doute et le potentiel entrepreneurial

\begin{tabular}{|c|c|c|c|}
\hline Variable de contrôle & Création par Opportunité & Création par défi \\
\hline Santé de l'entreprise & $\begin{array}{c}\text { Doute / envie d'aban- } \\
\text { donner }\end{array}$ &,$- 244^{* *}$ &,- 165 \\
\hline
\end{tabular}

Tableau 7. Corrélation partielle entre le doute et le potentiel entrepreneurial avec neutralisation de la santé de l'entreprise

\subsection{Résultats : des doutes au cœur de la relation entrepreneur-accompagnateur}

Les données quantitatives montrent clairement que lorsque les entrepreneurs doutent, autrement dit lorsqu'ils envisagent d'arrêter l'entreprise pour devenir salarié, la relation avec leur accompagnateur tend à se dégrader (tableaux 8 et 9). Ces données font également ressortir le rôle de la confiance au sein de cette dégradation : la confiance que les entrepreneurs accordent à leur suiveur, mais aussi la confiance qu'ils estiment que ce dernier leur accorde (tableaux 12 et 13).

\subsubsection{Des doutes à la dégradation de la relation entrepreneur-accompagnateur}

Les données du questionnaire montrent que le fait de douter et d'envisager d'arrêter s'accompagne souvent d'une dégradation de la relation, aussi bien dans ses dimensions techniques $(-0,28, p<0,00)$ que dans ses dimensions affectives $(-0,21 ; \mathrm{p}<0,02)$. On pourrait être tenté d'interpréter cette corrélation comme les effets d'une même variable, en l'occurrence la santé de l'entreprise, mais les corrélations partielles montrent qu'à santé égale, la corrélation avec l'efficacité de la relation demeure significative $(-0,218, \mathrm{p}<0,02)$. La corrélation partielle avec le caractère amical de la relation $(-0,17, \mathrm{p}<0,63)$ atteint un niveau de significativité proche du niveau requis $(\mathrm{p}<0,05)$. Le sens de la causalité reste à établir, mais on peut faire l'hypothèse que l'incertitude sur le fait de vouloir continuer rend la relation plus ambiguë, la remet en question et la dégrade.

\begin{tabular}{|c|c|c|}
\hline & Création / Opportunite & $\begin{array}{c}\text { Création } \\
\text { par défi }\end{array}$ \\
\hline Doute / envie d'abandonner & $-0,25^{* *}$ & $-0,18^{*}$ \\
\hline
\end{tabular}

Tableau 8. Corrélation entre le doute et l'évaluation de la relation 


\begin{tabular}{|c|c|c|c|}
\hline Variable de contrôle & Création par Opportunité & Création par défi \\
\hline Santé de l'entreprise & $\begin{array}{c}\text { Doute / envie d'aban- } \\
\text { donner }\end{array}$ &,$- 244^{* *}$ &,- 165 \\
\hline
\end{tabular}

Tableau 9. Corrélation partielle entre le doute et l'évaluation de la relation avec neutralisation de la santé de l'entreprise

La suite de cette série d'analyses a pour objectif de mieux comprendre les tenants et les aboutissants de la dégradation durant les phases de doute. Nous considérons que les facteurs qui jouent sur la relation ne sont pas forcément les mêmes lorsque les entrepreneurs persévèrent et lorsqu'ils envisagent d'arrêter. Suivant cette perspective, nous segmentons notre échantillon en ne gardant que les entrepreneurs ayant répondu positivement à la question 44 sur l'envie d'abandonner l'entreprise pour devenir salarié $(\mathrm{n}=$ 46).

\begin{tabular}{|c|c|c|c|c|c|c|}
\hline & $\begin{array}{c}\text { Difficultés } \\
\text { Perso }\end{array}$ & Tranquillité & Respecté & $\begin{array}{c}\text { Connaît un } \\
\text { créateur }\end{array}$ & $\begin{array}{c}\text { Confiance } \\
\text { Donnée }\end{array}$ & $\begin{array}{c}\text { Confiance } \\
\text { Reçue }\end{array}$ \\
\hline $\begin{array}{c}\text { Relation } \\
\text { efficace }\end{array}$ & $-0,51 * *$ & $0,39 *$ & $0,33 *$ & $0,39 * *$ & $0,79 * *$ & $0,57 * *$ \\
\hline
\end{tabular}

Tableau 10. Corrélation avec l'efficacité de la relation segment doute / envie d'abandonner

\begin{tabular}{|c|c|c|c|}
\hline Variable de contrôle & Création par Opportunité & Création par défi \\
\hline Santé de l'entreprise & $\begin{array}{c}\text { Doute / envie d'aban- } \\
\text { donner }\end{array}$ &,$- 244^{* *}$ &,- 165 \\
\hline
\end{tabular}

Tableau 11. Corrélation avec l'amitié de la relation segment doute / envie d'abandonner

L'efficacité de la relation semble très liée aux difficultés personnelles : le sens de cette causalité reste à établir, mais il semble y avoir un parallèle entre la dégradation des relations professionnelles avec les accompagnateurs et les dégradations souvent observées sur le terrain dans la vie personnelle de couple et de famille. Les entrepreneurs ne sont plus tranquilles, ne se sentent plus respectés, ce qui affecte la qualité de leurs relations avec les autres en général. Concernant le côté amical, la santé de l'entreprise semble aggraver les effets du doute et introduire un seuil au delà duquel «plus rien ne va », ajoutant, en plus des difficultés techniques, des tensions socio-affectives.

Dans l'efficacité comme dans l'amitié, la confiance joue un rôle important : la confiance donnée et la confiance reçue. Ces deux variables ne ressortaient pas dans les tables de corrélation établies à partir de l'ensemble de la population $(\mathrm{n}=127)$, elles semblent intervenir surtout dans le cadre du processus de dégradation de la relation caractérisant la partie de l'échantillon envisageant d'arrêter l'entreprise pour devenir salarié $(n=46)$. La dégradation de la relation affecte la confiance ce qui constitue une remise en cause plus profonde de la relation. On peut noter que la dégradation de l'efficacité touche plus la confiance accordée aux accompagnateurs alors que la dégradation de la relation affective implique la confiance que les entrepreneurs ont l'impression de recevoir.

Le fait de connaître quelqu'un ayant créé une entreprise semble modérer la dégradation de la relation tant dans ses aspects techniques que dans ses dimensions affectives. Nous pouvons supposer que cette expérience constitue pour les entrepreneurs un repère leur permettant de relativiser leur propre expérience, de comprendre que les difficultés rencontrées sont en quelque sorte normales.

Suivant une régression descendante, la connaissance, la confiance donnée et la confiance reçue, avec des beta standardisés respectivement égaux à 0,$3 ; 0,7$ et 0,2 , prédisent relativement bien le niveau d'efficacité de la relation ( $\mathrm{r}^{2}$ ajusté $\left.=0,78, \mathrm{~F}=42,8, \mathrm{p}<0,001\right)$. La régression sur la dimension amicale de la rela- 
tion est moins satisfaisante : les variables explicatives retenues en sélection descendante étaient la connaissance d'un créateur, la santé de l'entreprise, la confiance reçue. Les « beta » étaient égaux à 0,$21 ; 0,25$ et 0,34 mais seul le dernier avait un « $\mathrm{t}$ » significatif. La régression aboutit à un $\mathrm{r}^{2}$ ajusté de 0,26 associé à un $\mathrm{F}$ égal à 6,2 significatif à 0,001 .

\subsubsection{Des doutes à la dégradation de la confiance}

Compte tenu des tableaux 12, 13 et 14, la confiance donnée semble plutôt liée aux difficultés technicoéconomiques alors que la confiance reçue, plus précisément la confiance que les entrepreneurs pensent que leurs accompagnateurs leur accordent, semble plus complexe, mêlant les éléments techniques et affectifs de la relation. La confiance reçue semble liée à l'estime de soi : l'individu perd sa fierté et ne se sent pas respecté. L'estime de soi est une variable importante dans la construction de soi en interaction avec les autres. Ces problèmes semblent également affecter les relations personnelles des entrepreneurs.

\begin{tabular}{|c|c|c|c|c|}
\hline & $\begin{array}{c}\text { LOC } \\
\text { Interne }\end{array}$ & $\begin{array}{c}\text { Santé de l'entre- } \\
\text { prise }\end{array}$ & $\begin{array}{c}\text { Difficultés person- } \\
\text { nelles }\end{array}$ & $\begin{array}{c}\text { Relation } \\
\text { efficace }\end{array}$ \\
\hline Confiance donnée &, $409\left(^{* *}\right)$ &, $348(*)$ &,$- 425(* *)$ &, $794(* *)$ \\
\hline
\end{tabular}

Tableau 12. Corrélation avec la confiance donnée segment doute / envie d'abandonner

\begin{tabular}{|l|c|c|c|}
\hline & Connaît créateur & LOC Externe & Ddifficultés personnelles \\
\hline Confiance reçue &, $326^{*}$ &,$- 335^{*}$ &,$- 395^{* *}$ \\
\hline
\end{tabular}

Tableau 13. Corrélation avec la confiance reçue segment doute / envie d'abandonner

\begin{tabular}{|c|c|c|c|c|c|}
\hline & $\begin{array}{c}\text { Expérience } \\
\text { positive }\end{array}$ & $\begin{array}{c}\text { Se sent Respec- } \\
\text { té }\end{array}$ & Se sent Fier & $\begin{array}{c}\text { Relation Effi- } \\
\text { cace }\end{array}$ & $\begin{array}{c}\text { Relation Ami- } \\
\text { cale }\end{array}$ \\
\hline Confiance reçue &, $313^{*}$ &, $294^{*}$ &, $309^{*}$ &, $572 * *$ &, $454^{* *}$ \\
\hline
\end{tabular}

Tableau 14. Corrélation avec la confiance reçue segment doute / envie d'abandonner

\begin{tabular}{|c|c|}
\hline & Difficultés personnelles \\
\hline LOC Interne &,- 369 \\
\hline
\end{tabular}

Tableau 15. Corrélation entre la localisation des contrôles et l'attribution de difficultés personnelles

L'un des résultats les plus intéressants de cette recherche est le lien entre la localisation du contrôle et la dégradation de la relation. Les internes, ceux qui relient la réussite de l'entreprise à leurs qualités personnelles, ceux qui ne croient pas à la chance ${ }^{9}$, maintiennent une relation de confiance malgré les difficultés. A l'inverse, les externes, ceux qui imputent des conjonctures extérieures à leur conduite de l'entreprise, notamment des difficultés personnelles (tableau 15, -0,369, sig 0,053) ${ }^{10}$, semblent remettre en cause la relation avec leur accompagnateur : ils lui retirent leur confiance et l'accusent de faire de même. Ces deux logiques appellent des réactions différenciées et adaptées de la part de ce dernier.

Suivant une régression descendante, l'internalité $(0,37)$, les difficultés personnelles $(-0,22)$ et l'efficacité de la relation $(0,55)$ permettent de prédire de façon relativement sûre la confiance donnée $\left(\mathrm{r}^{2}=0,7 ; \mathrm{F}=\right.$ $30,1, p>0,001)$. Dans une moindre mesure, l'externalité $(-0,30)$, l'efficacité de la relation $(0,36)$, l'amitié $(0,30)$ et le sentiment d'être respecté $(0,30)$ constituent des indicateurs utiles pour connaître le niveau de la confiance reçue ( $\mathrm{r}^{2}$ ajusté $\left.=0,49 ; \mathrm{F}=9,9 \mathrm{p}>0,001\right)$. 


\section{Discussion}

Les données de cette recherche nous permettent de mieux comprendre ce qui se passe lorsque, face aux difficultés de l'entreprise, les entrepreneurs sont tentés d'abandonner. Elles nous permettent également de mieux de cerner les évolutions de la relation dans le cadre de laquelle les accompagnateurs tentent d'apporter leur soutien. Compte tenu de ces données, nous proposons ici un certain nombre de pistes d'action.

Les données qualitatives montrent que la plupart des entrepreneurs passent par des périodes de doute. Il y a toujours des difficultés (Boyd et Gumper,1983 ; Adebowale,1994 ; Afzalur, 1996 ; Cardon et McGrath, 1999), mais certaines fois, au lieu de répondre par de la persévérance (Afzalur, 1996 ; Cardon et McGrath, 1999), les entrepreneurs pensent à s'arrêter pour devenir salarié. Ce doute constitue, selon nous (Valéau, 2004), une étape du processus d'implication dans l'activité entrepreneuriale : après une phase d'engagement très intense dans l'action, le doute constitue un «lâcher prise " à travers lequel les entrepreneurs se remettent en question et actualisent leurs positions. Comme le montrent Piaget (1971) et Watzlavick (1975), le doute et la confusion sont un préalable au changement. Il importe de sensibiliser les accompagnateurs afin qu'ils puissent diagnostiquer ces remises en question, les distinguer des stress plus ordinaires. Durant ces phases de doute, leur rôle ne peut plus, selon nous, se limiter à une aide technique mais doit, d'une façon ou d'une autre, comprendre un soutien psychologique. Ce soutien constitue une condition importante pour que les entrepreneurs puissent développer à nouveau la vision de leur projet d'entreprise (Verstraete, 1997, Saporta et Verstraete) en lien avec leur projet personnel (Bruyat, 1994 ; Verstraete, 2002). A cette fin, les approches rogériennes reprises par les tenants du counseling (Priels, 2004) constituent une réponse possible : une écoute associée à une acceptation inconditionnelle, permet d'accepter le doute et favorise la reconstruction.

Le problème est que c'est précisément dans ces moments de doute, lorsque l'accompagnement psychologique est utile, voire nécessaire, qu'il devient plus compliqué à prodiguer. La relation entrepreneuraccompagnateur peut, en effet, suivre un cercle vicieux : plus les performances sont mauvaises, plus les entrepreneurs doutent, plus la relation leur semble inefficace, plus elle devient effectivement inefficace, plus les performances se détériorent. Dans ce contexte, les accompagnateurs risquent, s'ils n'y prennent garde, d'alimenter ce processus de dégradation soit en se remettant eux-mêmes en cause, soit en remettant en cause les entrepreneurs. Partant de là, il nous semble crucial que les accompagnateurs aient conscience de ce processus. Il leur faut accepter cette perturbation de la relation et les tensions qu'elle génère comme un élément de contexte en dépit duquel ils doivent aider et soutenir leurs interlocuteurs.

Nos résultats montrent que le découragement ne prend pas la même forme pour tous les entrepreneurs : les internes ne fonctionnent pas tout à fait de la même façon que les externes. La mise à jour de cet aspect de la personnalité des entrepreneurs permet à aux accompagnateurs d'adapter leur attitude :

face aux externes qui ne leur font plus confiance et les accusent de faire de même, les accompagnateurs ont tout intérêt à ne pas répondre pour ne pas rentrer en conflit, ils doivent rester centrés sur la santé de l'entreprise, cette variable étant la seule cause de dégradation de la relation sur laquelle ils puissent effectivement intervenir ;

- $\quad$ face aux internes qui, d'une façon générale, tendent à se remettre en cause, les accompagnateurs doivent accomplir un travail différent, à certains égards plus subtil : un travail impliquant les dimensions affectives de la relation au moins autant que ses aspects techniques. Il s'agira de renforcer l'estime de soi de ces entrepreneurs en faisant ressortir les succès, en leur renvoyant une image positive d'eux-mêmes, en leur manifestant plus ouvertement que d'ordinaire les signes de la confiance qu'ils leur accordent.

Nos données indiquent que le fait de connaître quelqu'un qui a créé une entreprise modère les effets des doutes sur la relation. Les entrepreneurs relativisent ainsi leurs difficultés : ils réalisent qu'elles ne sont pas propres à leur parcours, mais qu'elles font partie intégrante du processus entrepreneurial. Partant de là, il peut être utile de communiquer sur le caractère " normal » de l'épreuve. Il peut être intéressant de 
sensibiliser dès le départ les individus désireux de se lancer en affaires à l'expérience à venir, en les informant qu'ils passeront certainement par des moments de doute. Lorsque apparait ce dernier, il peut être utile de leur rappeler que la plupart des autres «passent par là », de leur dire qu'il convient d'attendre avant de prendre une décision définitive, de leur préciser que la plupart des entrepreneurs doutent puis repartent. La crédibilité et la légitimité de ces messages pourraient, par ailleurs, être accrues, s'ils étaient délivrés par d'autres entrepreneurs.

Enfin, nos analyses montrent que ceux qui ont choisi de monter leur entreprise pour saisir une opportunité ou par défi résistent mieux à l'adversité que les autres. Ce constat irait dans le sens d'une personnalité entrepreneuriale (Brockaus \& Horwitz, 1985 ; Johnson, 1990). Ce faisant, les accompagnateurs peuvent être tentés de sélectionner les individus les mieux disposés à l'exercice. Mais cette option recèle, selon nous, un paradoxe, une négation des dispositifs d'aide à la création d'entreprise : les « vrais » entrepreneurs ont, en quelque sorte, moins besoin d'accompagnement que les autres; ce sont précisément les individus les moins bien disposés qui ont le plus besoin de cette aide. Dans le contexte de chômage actuel, les pouvoirs publics cherchent à amener le plus grand nombre à la création d'entreprise, y compris des gens moins disposés. Suivant cette perspective, nous pensons qu'il s'agit d'apporter un appui technique, mais également un soutien psychologique, il s'agit d'aider ces individus à dépasser leurs doutes et leurs craintes pour les amener à devenir, chemin faisant, de « véritables » entrepreneurs.

\section{Conclusion}

La phase de doutes constitue un moment crucial de l'aventure entrepreneuriale. Au-delà de la santé économique des entreprises, elle touche le développement psychologique et social de ceux qui les impulsent. Durant cette période, les entreprises courent un double risque : tout d'abord les hésitations et la confusion qui caractérisent le doute apparaissent peu compatibles avec la détermination et la clairvoyance exigées par l'activité entrepreneuriale ; il reste, ensuite, la possibilité que les entrepreneurs décident d'y mettre définitivement fin. Ainsi, chaque année, des entreprises disparaissent par forfait, à cause d'un découragement prématuré, alors qu'elles auraient sans doute pu survivre.

Certains auteurs en concluront peut-être que ceux qui abandonnent n'étaient pas de " vrais » entrepreneurs. Au contraire, selon nous, le doute rapproche, à différents égards, les entrepreneurs des autres individus : ce doute, plus ou moins existentiel, face aux choix de la vie, constitue un phénomène humain relativement ordinaire qui, face aux exigences de l'aventure entrepreneuriale, prend une tournure encore plus déterminante. Après la création de l'entreprise, cette période de doute constitue une épreuve psychologique décisive à l'issue de laquelle l'apprenti-entrepreneur confirmera ou non son choix vocationnel.

Face à cette indétermination, les accompagnateurs peuvent, dans certains cas, faire la différence, en aidant les entrepreneurs à achever leur métamorphose, en sauvant ce faisant leur entreprise. Mais, les doutes des entrepreneurs exacerbent la complexité et l'ambiguïté de leur relation avec les accompagnateurs. Dans ce contexte, le soutien est nécessaire et en même temps difficile à prodiguer. Le doute est souvent lié à la santé de l'entreprise tout en impliquant des remises en question plus générales. Il touche les éléments techniques et les dimensions affectives de la relation. Suivant la personnalité des entrepreneurs, il se traduit plutôt par une remise en cause des accompagnateurs ou plutôt par une perte de confiance en soi. La plupart des accompagnateurs ressentent intuitivement ces moments de flottement et s'efforcent spontanément d'apporter leur soutien. Une connaissance plus approfondie de ces différents phénomènes liés à la phase de doute leur permettrait de mieux se positionner et d'aider plus efficacement les entrepreneurs.

Cet article contribue à cette connaissance, mais il garde, en dépit des éléments statistiques introduits, un caractère largement exploratoire. L'un des principaux défis à venir consistera à améliorer le passage de la richesse des explorations qualitatives aux mesures quantitatives. Nous avons, dans le cadre de cet arti- 
cle, faute de mieux, cantonné le doute à sa manifestation la plus directe : le fait d'envisager d'arrêter, que nous avons relié à différents autres indicateurs. Il reste encore à développer un construit plus abouti constitué de multiples items, à mieux cerner la complexité de ses relations avec les autres phénomènes impliqués, tout en intégrant le degré d'hésitation et de confusion global des entrepreneurs. A un autre niveau, cet article apporte un certain nombre de connaissances sur la relation entrepreneuraccompagnateur, mais celle-ci est abordée uniquement du point de vue des entrepreneurs ; il serait par la suite utile d'intégrer l'autre côté de l'histoire en recueillant le point de vue des accompagnateurs. Il s'agirait d'identifier les différentes réponses possibles de ce dernier face aux doutes de son interlocuteur et de mesurer leurs impacts sur la qualité et l'efficacité de la relation. Nous faisons ainsi l'hypothèse de la contingence : le succès de cette collaboration dépend sans doute de la compatibilité des styles. Suivant cette perspective, l'objet de recherche deviendrait la « dyade " (Valéau, 2004) entrepreneuraccompagnateur.

La recherche et la formation sur l'accompagnement des entrepreneurs durant les phases de doute seraient, selon nous, aussi utiles que celles concernant les structures financières et les stratégies de développement marketing. Un tel investissement pourrait, croyons nous, contribuer à une diminution significative des cessations d'entreprises. Dans la mesure où l'heure ne paraît plus être à la sélection naturelle des entrepreneurs, le défi semble désormais consister à amener un nombre de plus en plus important d'individus vers la création d'entreprise. Suivant cette perspective, il importe plus que jamais d'étudier comment on "devient » entrepreneur et de comprendre la place du doute dans ce processus, afin de mieux cerner l'aide que peuvent apporter les accompagnateurs.

\section{Notes}

$1 \mathrm{cf} 1.3$

2 Entretiens réalisés et retranscrits par la chaire entrepreneuriale du Pr LJ Filion

3 Cf. 1.2.

4 Cf. Yin, 1984 ; Guba \& Licoln, 1994 ; Glaser \& Strauss, 1967, Morse, 1994 ; Adler et Adler, 1994 ;

5 «When the going gets tough... toward a psychological of entrepreneurial failure and remotivation »

6 Le « durcissement » d'un champ scientifique correspond, pour Stengers (1987), à une phase de stabilisation des problématiques et de structuration des débats.

7 A propos de l'accompagnement vers l'insertion sociale et professionnelle.

8 A propos de l'accompagnement des projets de développement par les volontaires dans les ONG.

9 Compte tenu de la corrélation négative, moins on est externe, autrement dit plus on est interne, moins on remet en cause la relation.

10 Corrélation non significative relativement proche du seuil 0,05 


\section{Annexe : Questionnaire}

Bonjour. Nous faisons une étude sur l'ADIE : ses points forts, ses points faibles et les améliorations possibles de son travail. Vous avez collaboré avec l'ADIE, c'est pourquoi nous aimerions vous poser un certain nombre de questions. La plupart de ces questions concernent vos opinions : nous vous soumettons un certain nombre d'affirmations en vous demandant si vous êtes d'accord ou non. Nous vous demandons de répondre sur une échelle de 1 à 7 , le 1 correspondant à " pas du tout d'accord », le 7 signifiant que vous êtes " tout à fait d'accord ». Ce questionnaire devrait prendre une vingtaine de minutes.

1. Depuis combien de temps avez-vous créé votre entreprise ? _ - Mois

A. Vos expériences et vos motivations

2. Vous avez choisi ce secteur d'activité compte tenu de ?

o Votre expérience antérieure ou de votre formation dans ce métier

Des opportunités et d'un marché estimé porteur 1234567

Des conseils de relations professionnelles ou familiales 1234567

De vos motivations personnelles $\quad 1234567$

Autres (précisez)

3. Quel est votre niveau d'études ?

4. Combien d'années aviez-vous travaillé avant la création de l'entreprise ?

années

5. Quelle expérience aviez-vous de la création d'entreprise ?

o Vous êtes issu(e) d'une famille d'entrepreneurs oui non

- Un membre de votre famille a créé une entreprise oui non

- Quelqu'un que vous connaissez a créé une entreprise oui non

o Vous aviez déjà une activité informelle (travail au noir) oui non

- Vous aviez déjà créé une entreprise dans ce secteur oui non

- Vous aviez déjà créé une entreprise dans un autre secteur oui non

6. Quelles étaient vos compétences avant la création de votre entreprise ?

o Vous étiez compétent(e) sur le produit ou le service proposé 1234567

o Vous étiez compétent(e) en comptabilité / finances 1234567

o Vous étiez compétent(e) en vente 1234567

o Vous étiez compétent(e) en organisation 1234567

7. Pourquoi avez-vous souhaité créer votre entreprise ?

o par besoin / par nécessité $\quad 1234567$

o pour être mon propre chef 1234567

o pour mieux gagner ma vie $\quad 1234567$

o $\quad$ pour mieux être respecté(e) 1234567

o par défi / par jeu 1234567

- $\quad$ Autres (précisez)

8. Pour réussir une entreprise, pensez-vous que

o II faut beaucoup de chance 1234567

- II faut beaucoup de préparation 1234567

o II faut certaines qualités personnelles 1234567

o II faut connaître des gens influents 1234567

o II faut beaucoup d'efforts 1234567 


\section{B. Votre entreprise}

9. En plus de vous-même, combien de personnes votre entreprise emploie-t-elle ?

o De manière permanente (toute l'année): _ _ _ personnes

o De manière occasionnelle ou saisonnière: $\quad-\quad \bar{p}-\overline{\text { rssonnes }}$

10. Globalement la santé de votre entreprise vous semble-t-elle bonne ? 1234567

11. Quel est le montant de vos ventes annuelles ? (chiffre d'affaires) (Ne pas citer les réponses dès le départ, ne les citer que si la personne ne sait que répondre précisément):

- Inférieur à $30000 \mathrm{FF}$ (4500 euros)

o $\quad$ Entre $30000 \mathrm{FF}$ et $50000 \mathrm{FF}$ (7500 euros)

o Inférieur à $100000 \mathrm{FF}$ (15000 euros)

- $\quad$ Entre 100000 et 200000 FF (30000 euros)

o $\quad$ Entre 200000 et 300000 FF (45000 euros)

o $\quad$ Entre 300000 et $400000 \mathrm{FF}$ (60000 euros)

o $\quad$ Entre 400000 et $500000 \mathrm{FF}$ (75000euros)

- Supérieur à 500000 FF (75000 euros)

- Ne sait pas

12. Ces ventes sont-elles supérieures à ce que vous aviez prévu ? 1234567 (avec 4 = comme vous aviez prévu)

13. Depuis la création de votre entreprise, ces ventes ont-elles progressé ? (avec 4 = stable ou irrégulier)

14. Actuellement, quels types de difficultés rencontrez-vous dans votre entreprise ?

o Des difficultés financières / de trésorerie 1234567

o $\quad$ Des problèmes de vente 1234567

o $\quad$ Des problèmes d'approvisionnement 1234567

- Des problèmes d'organisation 1234567

o Des difficultés personnelles qui interfèrent dans votre entreprise

Autres (précisez)

15. Selon vous, ces difficultés sont-elles durables? $\quad 1234567$

16. Quelle somme prélevez-vous en moyenne chaque mois sur les revenus de votre entreprise à titre de salaire ? (Ne pas citer les réponses dès le départ, ne les citer que si la personne ne sait que répondre ou pour faire préciser une réponse):

o Inférieure à $2000 \mathrm{FF}$ (300 euros)

o $\quad$ Entre 2000 et 3000 FF (450 euros)

- $\quad$ Entre 3000 et 4000 FF (600euros)

o $\quad$ Entre 4000 et $5000 \mathrm{FF}$ (750 euros)

o $\quad$ Entre 5000 et 7000 FF (1050 euros)

o $\quad$ Entre 7000 et $10000 \mathrm{FF}$ (1500 euros)

- Supérieure à 10000 FF (1500 euros)

- Ne sait pas

17. Est-ce suffisant pour faire face aux besoins de votre ménage ? 1234567

18. Disposez-vous d'autres revenus pour subvenir aux besoins de votre ménage ?

o Aucun

- Revenu du conjoint

- Indemnité ASSEDIC ou RMI

- Autre travail

o $\quad$ Autres (précisez) 
20. Globalement, les revenus de votre ménage vous permettent-ils d'économiser ou de cotiser à une mutuelle ou à une retraite complémentaire ? $\quad 1234567$

21. Avec la création de votre entreprise, votre situation financière s'est-elle améliorée ?

1234567

22. Diriez-vous que cette évolution de votre situation financière est liée à la création de votre entreprise? $\quad 1234567$

\section{Les prêts dont vous avez bénéficié}

23. Comment avez-vous connu l'ADIE (Ne pas citer les réponses dès le départ, ne les citer que si la personne ne sait que répondre)?

o Chambres consulaires

o $\quad$ Autres structures d'appui à la création d'entreprise (BG, PFIL etc.)

- Assistantes sociales

o DDTE

o Presse

- Amis, relations

- Un créateur ayant déjà bénéficié d'un prêt de l'ADIE

- $\quad$ Autres (précisez)

24. Vous avez pris contact avec l'ADIE ?

o Pour obtenir un crédit 1234567

- Pour pouvoir bénéficier de conseils et de suivi

1234567

25. J'aimerais maintenant que vous me donniez votre avis sur différents aspects du (ou des) crédits que vous avez obtenu auprès de l'ADIE.

- Avez-vous trouvé le montant du ou des prêts suffisant?

o Avez-vous reçu l'argent à temps ?

1234567

o Le montant des remboursements vous a-t-il semblé raisonnable?

o $\quad$ L'exigence du premier remboursement est-elle venue au bon moment ?

1234567

26. Je vais vous citer maintenant un certain nombre de services bancaires et vous demander pour chacun si vous en disposiez avant la création de l'entreprise et si vous en disposez aujourd'hui.

\begin{tabular}{|c|c|c|c|c|}
\hline & \multicolumn{2}{|c|}{ Avant la création } & \multicolumn{2}{|c|}{ Aujourd'hui } \\
\hline Compte courant personnel & oui & non & oui & non \\
\hline Compte épargne ou CODEVI & oui & non & oui & non \\
\hline Compte professionnel & oui & non & oui & non \\
\hline Chéquier & oui & non & oui & non \\
\hline Carte de retrait & oui & non & oui & non \\
\hline Carte de crédit & oui & non & oui & non \\
\hline Autorisation de découvert & oui & non & oui & non \\
\hline
\end{tabular}

27. Avez-vous sollicité un prêt bancaire pour votre entreprise depuis sa création ? Oui (Passez à la question 28) Non (Passez à la question 31) 
28. Le prêt que vous avez sollicité était-il destiné à

o Augmenter votre fonds de roulement (Trésorerie/stock)?

- Réaliser de nouveaux investissements (Développement)?

29. Avez-vous obtenu ce prêt?

Oui Non pourquoi _ _ _ _ _ (passer à la question 31)

30. Pourquoi (1 seule réponse) ?

- Vous n'en avez pas eu besoin

o Vous ne souhaitiez pas vous endetter

- Vous n'avez pas osé

o Vous étiez sûr(e) que vous ne l'obtiendriez pas

31. Avez-vous demandé une prime?

Non (Passez à la question 35)

Oui : Région /_/ Département /_I Autres

32. Avez-vous obtenu cette prime?

Non (Passez à la question 35)

Oui : Région /_/ Département /_I Autres

33. Auriez-vous créé votre entreprise s'il n'y avait pas eu cette prime?

34. Avez-vous reçu cette prime assez tôt?

1234567

35. Savez-vous s'il est possible pour vous de solliciter l'ADIE pour un nouveau prêt? Oui Non

36. Si cela est était possible, feriez-vous une nouvelle demande?

D.Le suivi dont vous avez bénéficié

37. Le suivi dont vous avez bénéficié a-t-il été assuré principalement assuré par?

o Un conseiller permanent de ADIE?

o Un bénévole de l'ADIE?

o Une autre structure (BG etc.) ?

38. En général, comment s'est déroulé le suivi ?

- Par des rencontres au bureau de l'ADIE

- Par des rencontres à votre domicile

- Par des rencontres sur votre lieu de travail

- Par des appels téléphoniques

39. A quelle fréquence avez-vous rencontré la personne qui vous a suivi ?

- Plusieurs fois par semaine

- Une fois par semaine

- Une fois tous les 15 jours

o Une fois par mois

- Une fois tous les deux mois

- Une fois par trimestre

- Moins d'une fois par trimestre 
40. La question suivante concerne l'aide dont vous aviez besoin et l'aide dont vous avez bénéficié dans différents domaines

\begin{tabular}{|l|c|c|}
\hline & Besoin d'aide & Qualité de l'aide obtenue \\
\hline Comptabilité/ finances & 1234567 & 1234567 \\
\hline Techniques de vente & 1234567 & 1234567 \\
\hline Administratif et juridique & 1234567 & 1234567 \\
\hline $\begin{array}{l}\text { Aide à la décision (stratégie, } \\
\text { investissement, etc.) }\end{array}$ & 1234567 & 1234567 \\
\hline Relation avec la banque & 1234567 & 1234567 \\
\hline Soutien moral & 1234567 & 1234567 \\
\hline $\begin{array}{l}\text { Autre (à préciser) } \\
-------\end{array}$ & 1234567 & 12367 \\
\hline
\end{tabular}

41. Comment voyez-vous votre relation avec votre suiveur?

o $\quad$ Diriez-vous que votre relation était efficace 1234567

o $\quad$ Diriez-vous que votre relation était amicale 1234567

- Diriez-vous que vous aviez confiance dans votre suiveur 1234567

o Pensez-vous que votre suiveur avait confiance en vous 1234567

\section{E. Conclusion}

42. La création de votre entreprise a-t-elle finalement été une expérience positive ? 1234567

- La création d'entreprise vous a-t-elle donné plus confiance en vous ? 1234567

- La création d'entreprise vous a-t-elle rendu(e) plus optimiste ? 1234567

o La création d'entreprise vous a-t-elle tranquillisé(e) ? 1234567

o La création d'entreprise vous a-t-elle rendu(e) plus entreprenant(e) ? 1234567

- La création d'entreprise a-t-elle rendu votre vie plus intéressante ?

o La création d'entreprise vous a-t-elle permis d'être mieux respecté(e) ? 1234567

43. Aujourd'hui, pensez-vous :

o Je suis fier(fière) d'avoir créé mon entreprise1 234567

- $\quad$ Arrêter mon entreprise aurait plus d'inconvénients que d'avantages 1234567

- Je continue mon entreprise parce que je ne vois pas d'autres possibilités 1234567

o Je me sens affectivement attaché(e) à mon entreprise 1234567

o II ne serait pas bien d'arrêter mon entreprise, même si j'y trouvais avantage 1234567

44. Pour l'avenir, préféreriez-vous (re)trouver un emploi salarié ? $\quad 1234567$ 


\section{Bibliographie}

ADEBOWALE A. (1994), « Coping with Entrepreneurial Stress », Journal of Small Business Management, 32(1).

ADLER P.A. \& ADLER P. (1994), « Observational Techniques ». in Denzin N. K. \& Lincoln S. Y., Handbook of Qualitative Research, Sage.

AFZALUR R. (1996), « Stress, Strain, and Their Moderators: An Empirical Comparison of Entrepreneurs and Managers », Journal of Small Business Management, 34(1).

ALLEN N.J. \& MEYER J.P. (1997), Commitment in the workplace: Theory, research, and application, Sage.

ALVAREZ J.L. (1993), "The diffusion and institutionalization of entrepreneurship education in the eighties », in Birley S. \& Macmillian I.C. Entrepreneurship research : global perspectives.

BARES R. \& MULLER F. (2002), « Dépasser les freins au soutien entrepreneurial », 2ème congrès de l'Académie de l'Entrepreneuriat, Bordeaux.

BEAUVOIS J.L. JOULE R.V. (1987), Petit traité de manipulation à l'usage des honnêtes gens, Presses Universitaires de Grenoble.

BECHARD J.P. (1998), « L'enseignement en entrepreneurship à travers le monde », Management International, 3(1).

BOYD D.P. \& GUMPER D.E. (1983), « The Effects of Stress on Early-Stage Entrepreneurs », Frontiers of Entrepreneurship Research.

BROCKHAUS R.H. \& HORWITZ P.S. (1985), «The Psychology of the Entrepreneur », in Sexton D.L. \& Smilor R.W., The Art and Science of Entrepreneurship, Ballinger.

BRUYAT C. (1994), «Contributions épistémologiques au domaine de l'entrepreneuriat », Revue Française de Gestion, 101.

BYGRAVE W.D. \& HOFER C.W. (1991), « Theorizing about entrepreneurship », Entrepreneurship Theory \& Practice, 16(2).

CANTILLON R (1957), Entrepreneur and Economist, Oxford, 1986.

CARDON G. \& McGrath R. (1999), « When the Going Gets Tough... Toward Psychology of Entrepreneurial Failure and Re-motivation », Frontiers of Entrepreneurship Research.

CARLAND J.W., HOY F., BOULTON W.R. \& CARLAND J.C. (1984), « Differentiating entrepreneurs from small Business owners », Academy of Management Review, 9(2).

CASTRA D. (2003), L'insertion professionnelle des publics précaires, Presses Universitaires de France.

CULLIERE O. (2004), « La légitimité du conseil en management au TPE », 7ème congrès CIFEPME, Montpellier.

DAVIDSON P. \& WIKLUND J. (2001), « Level of analysis in entrepreneurship research : current research practice and suggestions for the future », Entrepreneurship Theory \& Practice, 25(4). 
DAVIDSON P., LOW M.B., WRIGHT M. (2001), « Low and McMillan ten years on : achievements and future directions for entrepreneurship research », Entrepreneurship Theory \& Practice, 25(4).

DOKOU K.G. (2002), « De l'accompagnement du créateur de PME à l'exploitation dynamique des marchés », XIème Conférence Internationale ESCP-EAP.

DOKOU K.G., GASSE Y., ABIASSI A., CAMION C. (2004), «Influence des pratiques d'accompagnement et de recherche d'informations sur la stratégie des entrepreneurs de PME », Université de Laval, Document de travail 2004-001.

FILION L.J. (1997), « Le champ de l'entrepreneuriat », Revue Internationale PME, 10(2).

GARTNER W.B. (1988), "Who is an Entrepreneur is the wrong question », American Small Business Journal, Spring.

GARTNER W.B. \& GATEWOOD E. (1992), « Thus the theory of description matters most, Entrepreneurship Theory \& Practice, 17(1).

GARTNER WB, BIRD BJ, STARR JA (1992), « Acting as if : differentiating entrepreneurial from organizational behavior », Entrepreneurship Theory \& Practice 16(3).

GARTNER W.B., SHAVER K.G., GATEWOOD E., KATZ J.A., (1994), « Finding the entrepreneur in entrepreneurship », Entrepreneurship Theory \& Practice 18(3).

GLASER B. \& STRAUSS A. (1967), The discovery of grounded theory, strategies of qualitative research, Aldine Publishing Company.

GUBA E.G. ET LINCOLN Y.S. (1994), « Competing paradigms in Qualitative Research », in Denzin NK et Lincoln SY, Handbook of Qualitative Research, Sage.

JOHANNISSON B. (1991), « University training for entrepreneurship : a swedish approach », Entrepreneurship and Regional Development, 3(1).

JOHNSON, B. R. (1990), « Toward a multidimensional model of entrepreneurship: The case of achievement motivation and the entrepreneur », Entrepreneurship Theory and Practice, 14(3).

KIRZNER I.M. (1993), «The morality of Pure Profit », Journal des Economistes et des Etudes Humaines, 4.

KNIGHT F.H. (1921), Risk, Uncertainty and profit, Houghton Mifflin.

MILL J.S. (1848), Principle of Political Economy with some Applications to Social Philosophy, J.W. Parker.

NAFFZIGER D.W., HORNSBY J.S. \& KURATO D.F. (1994), « A proposed research model of entrepreneurial motivation», Entrepreneurship Theory \& Practice, 18(3).

PAILOT P. (2003), « Méthode biographique et entrepreneuriat : application à l'étude de la socialisation anticipée », Revue de l'Entrepreneuriat, 2(1).

PASQUIER D. \& LUCOT J.C. (1999), "Une nouvelle échelle de localisation du contrôle - interne externe », Pratiques Psychologiques, 2. 
PAULHUS D. (1984). «Two-component models of socially desirable responding », Journal of Personnality and Social Psychology, 46.

PIAGET J. (1971), «Inconscient affectif et inconscient cognitif », Raison Présente, 19.

PRIEL J.M. (2004), « Eléments historiques à propos du counseling ", Association Française de Counseling dans l'Approche Centrée sur la Personne, (http://www.geocities.com/afcacp).

ROGERS C. R. (1961), On becoming a person, Houghton Miffin Company.

SAMMUT S. (2003), "L'accompagnement des petites entreprises en création » in MARION S. ; NOEL X. ; SAMMUT S. SENICOURT P., Réflexion sur les outils et les méthodes à l'usage du créateur d'entreprise, Les Editions de l'ADREG (http://www.adreg.net).

SAPORTA B. ; VERSTRAETE T. (2000), Réflexions sur l'enseignement de l'entrepreneuriat, Gestion $2000,3$.

SAY J.B. (1803), Traité d'économie politique.

SCHUMPETER J.A. (1935), Théorie de l'évolution économique, Dalloz.

SHANE S. ; VENKATAMARAN S. (1997), « The promise of entrepreneurship as a field of research», Academy of Management Review, 25(1).

SHAVER K.G. (1995), "The entrepreneurial personality myth », Business and Economic Review, April-June.

SIMON M., HOUGHTON S.M., AQUINO M. (2000), « Cognitive biases, risk perception and venture formation », Journal of Business Venturing, 14(5).

STAW B.M . (1976), « Knee-deep in the big muddy », Organizational Behavior and Human Performance, 76.

STENGERS I. (1987), D'une science à l'autre des concepts nomades, Seuil.

STEVENS W.S. (1991), « Recharge your entrepreneurial batteries », Professional Press US.

UCBASARAN D., WESTHEAD P., WRIGHT M. (2001), " The focus of entrepreneurial research : contextual and process issues », Entrepreneurship Theory \& Practice, 25(4).

VALEAU P. (2004), Gérer l'implication dans le respect des différences, Habilitation à Diriger les Recherches, Lille.

VALEAU P., CIMPER P., FILION L.J. (2004), « Entrepreneuriat et organisations à but non lucratif », Cahier de recherche Chaire Rogers Bombardier, 2004-10.

VALEAU P. (2001), « Pour une version non seulement lucrative de l'entrepreneuriat, des exemples de la Zone Océan Indien », Management International, 6(1).

VERSTRAETE T. (2003), Proposition d'un cadre théorique pour la recherche en entrepreneuriat, Les Editions de l'ADREG (http://www.adreg.net).

VERSTRAETE T. (2002), Essai sur la singularité de l'entrepreneuriat comme domaine de recherche, Les Editions de l'ADREG (http://www.adreg.net). 
VERSTRAETE T. (1999), Entrepreneuriat : connaître l'entrepreneur, comprendre ses actes, L'Harmattan.

VERSTRAETE T. (1997), « Cartographie cognitive et accompagnement du créateur d'entreprise », Revue Internationale PME, 10(1).

VESALAINEN J. \& PIHKALA T. (1999), « Motivation Structure and Entrepreneurial Intention », Frontiers of Entrepreneurship Research, Babson College.

WACHEUX F. (1996), Méthodes qualitatives et recherche en gestion, Economica.

WATZLAVICK P. (1975), Changement, paradoxes et psychothérapie, Seuil, Point Essais.

WEBER M. (1921), Economy and Society. New York Bedminster Press.

WEICK K.E. (1979), The Social Psychology of Organizing, Addison-Westley.

YIN R.K. (1984), Case study research: Design and methods, Sage. 